CLNS 04/1898

hep-ph/0411027

November 1, 2004

\title{
Impact of Four-Quark Shape Functions on Inclusive B Decay Spectra
}

\author{
Matthias NeuberT \\ Institute for High-Energy Phenomenology \\ Newman Laboratory for Elementary-Particle Physics, Cornell University \\ Ithaca, NY 14853, U.S.A. \\ and \\ School of Natural Sciences, Institute for Advanced Study \\ Princeton, NJ 08540, U.S.A.
}

\begin{abstract}
It has recently been pointed out that a new class of subleading shape functions involving $B$-meson matrix elements of non-local four-quark operators contributes at order $\Lambda_{\mathrm{QCD}} / m_{b}$ to $\bar{B} \rightarrow X_{u} l^{-} \bar{\nu}$ decay distributions in the endpoint region. The corresponding functions $f_{u}(\omega)$ and $f_{v}(\omega)$ are estimated using the vacuum-insertion approximation. A numerical analysis of various $\bar{B} \rightarrow X_{u} l^{-} \bar{\nu}$ decay spectra suggests that these power corrections are very small, below present theoretical uncertainties due to other subleading shape-function contributions.
\end{abstract}


Inclusive decays of $B$ mesons into final states containing light particles, such as $\bar{B} \rightarrow X_{u} l^{-} \bar{\nu}$ and $\bar{B} \rightarrow X_{s} \gamma$, play an important role in the extraction of the element $\left|V_{u b}\right|$ of the quark mixing matrix. Experimental cuts in the analysis of these processes restrict the hadronic final state to have large energy, $E_{X} \sim m_{B}$, but only moderate invariant mass, $M_{X} \sim \sqrt{m_{B} \Lambda_{\mathrm{QCD}}}$. In this region of phase space, the inclusive rates can be calculated using a twist expansion, which resums infinite sets of local-operator matrix elements into non-perturbative shape functions [1, 2]. It is well known that the leading term in this expansion obeys a QCD factorization formula [3], which separates contributions associated with the hard scale $m_{b}$, the jet scale $\sqrt{m_{b} \Lambda_{\mathrm{QCD}}}$, and the soft scale $\Lambda_{\mathrm{QCD}}$. In recent work, the calculation of next-to-leading perturbative corrections to the various components in this formula has been completed [4, 5].

Remarkably, for the case of inclusive decay distributions QCD factorization can be extended beyond the leading order in the heavy-quark expansion. Using the formalism of soft-collinear effective theory (SCET) 6, 7], it has been argued that a factorization theorem holds at every order in $\Lambda_{\mathrm{QCD}} / m_{b}$ [8, 9]. Inclusive decay spectra therefore provide an example of a class of observables which have a systematic expansion in non-local string operators, built out of quark and gluon fields with light-like separation connected by Wilson lines. Non-perturbative hadronic physics is encoded in forward $B$-meson matrix elements of these operators in heavyquark effective theory (HQET) [10]. The expansion in string operators is a generalization of the conventional (local) operator product expansion for correlation functions at large (Euclidean) momentum transfer.

Recently, two groups have presented the first complete analyses of subleading power corrections to arbitrary $\bar{B} \rightarrow X_{u} l^{-} \bar{\nu}$ decay distributions in the shape-function region [8, 9]. In these studies, the QCD current-current correlator, whose forward $B$-meson matrix element is related to the hadronic tensor $W^{\mu \nu}$, is matched onto correlation functions in SCET. These correlators are then expanded in terms of non-local string operators in HQET. The resulting expressions generalize (and in some cases correct) previous results in the literature [11, 12, 13. In particular, it was pointed out that there exist some tree-level contributions involving subleading shape functions defined in terms of non-local four-quark operators, which had not been considered previously. Their contributions to arbitrary decay distributions can be parameterized in terms of two functions $f_{u}(\omega)$ and $f_{v}(\omega)$ defined as 9 ]

$$
\int d \omega e^{-i \omega t}\left[f_{u}(\omega) T_{1}+f_{v}(\omega) T_{4}\right]=(-i)^{2} \int_{0}^{t} d t_{1} \int_{t_{1}}^{t} d t_{2} \frac{\left\langle\bar{B}(v)\left|O_{4 q}\left(t_{1}, t_{2}, t\right)\right| \bar{B}(v)\right\rangle}{2 m_{B}}
$$

where

$$
O_{4 q}\left(t_{1}, t_{2}, t\right)=(\bar{h} S)_{0} \Gamma_{i} \not h \gamma_{\rho}^{\perp} t_{A}\left(S^{\dagger} q\right)_{t_{1} n}(\bar{q} S)_{t_{2} n} \gamma_{\perp}^{\rho} \not h \Gamma_{j} t_{A}\left(S^{\dagger} h\right)_{t n}
$$

is a non-local string operator with fields ordered along the light-cone defined by a vector $n$. Here $h$ is a heavy-quark field in HQET, $q$ is a massless quark ( $q=u$ for semileptonic decay), $S$ denotes a soft Wilson line, and $t_{A}$ are the generators of color $\mathrm{SU}\left(N_{c}\right)$. The light-like vector $n$ points in the direction of the final-state hadronic jet $X_{u}$. We work in the $B$-meson rest frame, where $v^{\mu}=(1,0,0,0)$, and take $n^{\mu}=(1,0,0,1)$. Perpendicular Lorentz indices refer to the transverse plane orthogonal to $v$ and $n$ (see 9] for further definitions and notation). The traces

$$
T_{1}=\frac{1}{4} \operatorname{tr}\left[\Gamma_{i} \not h \Gamma_{j} \frac{1+\psi}{2}\right], \quad T_{4}=\frac{1}{4} \operatorname{tr}\left[\Gamma_{i} \not h \gamma_{5} \Gamma_{j} \frac{1+\psi}{2}(\psi-\not h) \gamma_{5}\right]
$$


depend on the Dirac structures of the flavor-changing weak currents $J_{i}^{\dagger}=\bar{b} \Gamma_{i} q$ and $J_{j}=\bar{q} \Gamma_{j} b$ in the definition of the hadronic tensor, and $\Gamma_{i}, \Gamma_{j}$ are in principle arbitrary Dirac matrices. Expanding both sides of (10) in powers of $t$, one finds that the functions $f_{u}$ and $f_{v}$ have zero norm and first moment, whereas their second moments are given in terms of a local four-quark matrix element,

$$
\int d \omega \omega^{2}\left[f_{u}(\omega) T_{1}+f_{v}(\omega) T_{4}\right]=\frac{\left\langle\bar{B}(v)\left|\bar{h} \Gamma_{i} \not h \gamma_{\rho}^{\perp} t_{A} q \bar{q} \gamma_{\perp}^{\rho} \not h \Gamma_{j} t_{A} h\right| \bar{B}(v)\right\rangle}{2 m_{B}} .
$$

The subleading shape functions $f_{u}$ and $f_{v}$ enter the decay rates multiplied by a factor $\pi \alpha_{s}\left(\mu_{i}\right) \approx$ 1 , where $\mu_{i} \sim \sqrt{m_{b} \Lambda_{\mathrm{QCD}}}$ is an intermediate matching scale. Because of the factor $\pi$, this perturbative coupling does not provide a numerical suppression.

While the authors of [8, 9] agree on the structural form of the four-quark contributions, they differ in the assessment of the expected numerical importance of their effects. In [9], it is argued that the four-quark contributions are expected to be suppressed with regard to other subleading shape functions. On the other hand, the authors of [8] employ arguments based on naive dimensional analysis (including counting factors of 4) to speculate that these effects may be the dominant source of $\Lambda_{\mathrm{QCD}} / m_{b}$ corrections, which could lead to $O(1)$ effects in some decay spectra. In light of this controversy, it may be of some value to have well-motivated, if model-dependent estimates of the subleading shape functions $f_{u}$ and $f_{v}$. This is what we will provide in this Letter.

We first consider the case where the flavor of the light quark $q$ matches that of the $B$ meson spectator quark. It is empirically well established that the magnitude of forward $B$-meson matrix elements of local four-quark operators can be estimated by inserting the vacuum intermediate state, thereby factorizing them into products of current matrix elements. This approximation is routinely used, e.g., in the analysis of lifetime ratios of beauty hadrons [14, 15]. Depending on the color structure of the operators, it is conventional to define $\left\langle\bar{B}_{q}\left|\bar{h} \Gamma_{1} q \bar{q} \Gamma_{2} h\right| \bar{B}_{q}\right\rangle \equiv \mathcal{B}_{i}\left\langle\bar{B}_{q}\left|\bar{h} \Gamma_{1} q\right| 0\right\rangle\left\langle 0\left|\bar{q} \Gamma_{2} h\right| \bar{B}_{q}\right\rangle$ and $\left\langle\bar{B}_{q}\left|\bar{h} \Gamma_{1} t_{A} q \bar{q} \Gamma_{2} t_{A} h\right| \bar{B}_{q}\right\rangle \equiv$ $\varepsilon_{i}\left\langle\bar{B}_{q}\left|\bar{h} \Gamma_{1} q\right| 0\right\rangle\left\langle 0\left|\bar{q} \Gamma_{2} h\right| \bar{B}_{q}\right\rangle$, where the subscript " $i$ " refers to different Dirac structures. The large- $N_{c}$ counting rules of QCD imply $\mathcal{B}_{i}=O(1)$ and $\varepsilon_{i}=O\left(1 / N_{c}\right)$, and this hierarchy is preserved under renormalization. Theoretical work based on lattice QCD [16, 17] and QCD sum rules [18] suggests that the $\varepsilon_{i}$ parameters are indeed rather small, typically of order 0.1 or less. (More specifically, [17] quotes $\varepsilon_{1} \approx \varepsilon_{2} \approx 0.01$ at a scale of $2.7 \mathrm{GeV}$, whereas [18 finds $\varepsilon_{1} \approx-0.04$ and $\varepsilon_{2} \approx 0.06$ at a scale of $1 \mathrm{GeV}$.) This is consistent with empirical findings. For instance, at a renormalization point $\mu \approx m_{b} / 2$, the lifetime ratio of charged and neutral $B$ mesons can be written as 15

$$
\frac{\tau\left(B^{+}\right)}{\tau\left(B^{0}\right)} \approx 1+0.044 B_{1}+0.003 B_{2}-0.74 \varepsilon_{1}+0.20 \varepsilon_{2} \stackrel{!}{=} 1.086 \pm 0.017,
$$

where the last result is the current experimental value. If the $\varepsilon_{i}$ parameters were much larger than of order 0.1 , a fine tuning would be required in order to avoid a large deviation from the experimental value. On the other hand, with $B_{i} \approx 1$ and $\varepsilon_{i}=O(0.1)$ it is easy to reproduce the experimental result. 
There appears to be no reason why color suppression should be less effective for non-local four-quark operators than for local ones. As a model, we are thus led to replace

$$
\begin{aligned}
\left\langle\bar{B}_{q}(v)\left|O_{4 q}\left(t_{1}, t_{2}, t\right)\right| \bar{B}_{q}(v)\right\rangle & \approx \varepsilon\left\langle\bar{B}_{q}(v)\left|\bar{h}(0)\left[0, t_{1}\right] \Gamma_{i} \not h \gamma_{\rho}^{\perp} q\left(t_{1} n\right)\right| 0\right\rangle \\
& \times\left\langle 0\left|\bar{q}\left(t_{2} n\right)\left[t_{2}, t\right] \gamma_{\perp}^{\rho} \not h \Gamma_{j} h(t n)\right| \bar{B}_{q}(v)\right\rangle,
\end{aligned}
$$

where $\left[t_{k}, t_{l}\right]$ denotes a straight soft Wilson line connecting the points $t_{k} n$ and $t_{l} n$, and $\varepsilon$ is the color-suppression factor. This ansatz completely specifies our model for the subleading shape functions. Whereas the definitions of the parameters $\mathcal{B}_{i}$ and $\varepsilon_{i}$ introduced in [15] are completely general, the introduction of the parameter $\varepsilon$ in the equation above corresponds to a model hypothesis, because we assume that $\varepsilon$ is independent of the position arguments $t_{1}$, $t_{2}, t$ and of the Dirac structures $\Gamma_{i}, \Gamma_{j}$. Nevertheless, as we shall see, our model provides an expression for the subleading shape functions $f_{u}$ and $f_{v}$ with all the right properties such as the correct support and moment relations, as far as they are known. We expect that it predicts the rough overall scale of the effect reliably. In fact, many analyses of inclusive $B$ decays are based on measurements of partial decay rates over kinematical domains that fall in between the shape-function region and the region where a conventional operator product expansion can be applied [5]. In such a situation, the dominant contributions to the decay rates are associated with the lowest non-zero moments of the shape functions. In the case of the fourquark shape functions, the lowest non-zero moments are given by the matrix element of the local four-quark operator in (4), for which the vacuum insertion approximation is certainly reasonable.

The $B \rightarrow$ vacuum matrix elements of the non-local quark bilinears in (6) can be expressed in terms of the leading light-cone distribution amplitude of the $B$ meson [19],

$$
\left\langle 0\left|\bar{q}\left(t^{\prime} n\right)\left[t^{\prime}, t\right] \not h \Gamma h(t n)\right| \bar{B}_{q}(v)\right\rangle=-\frac{i f_{B} m_{B}}{2} \operatorname{tr}\left[h \Gamma \frac{1+\psi}{2} \gamma_{5}\right] \int_{0}^{\infty} d \omega \phi_{+}(\omega) e^{-i \omega t^{\prime}-i(\bar{\Lambda}-\omega) t},
$$

where we have used that the $B$ meson in HQET carries momentum $\bar{\Lambda} v$, with $\bar{\Lambda}=m_{B}-m_{b}$. Throughout this note we work at lowest order in perturbation theory, so that we can ignore the scale dependence of the various objects in the above equation. Taking into account that the distribution amplitude $\phi_{+}$is real, we obtain in our model

$$
\begin{aligned}
& (-i)^{2} \int_{0}^{t} d t_{1} \int_{t_{1}}^{t} d t_{2}\left\langle\bar{B}_{q}(v)\left|O_{4 q}\left(t_{1}, t_{2}, t\right)\right| \bar{B}_{q}(v)\right\rangle \\
& =\varepsilon \frac{f_{B}^{2} m_{B}^{2}}{4} e^{-i \bar{\Lambda} t} \operatorname{tr}\left[\gamma_{5} \frac{1+\psi}{2} \Gamma_{i} \not h \gamma_{\rho}^{\perp}\right] \cdot \operatorname{tr}\left[\gamma_{\perp}^{\rho} \not h \Gamma_{j} \frac{1+\psi}{2} \gamma_{5}\right] \\
& \times \int_{0}^{\infty} d \omega_{1} \int_{0}^{\infty} d \omega_{2} \phi_{+}\left(\omega_{1}\right) \phi_{+}\left(\omega_{2}\right)\left[\frac{1}{\omega_{1} \omega_{2}}+\frac{1}{\omega_{1}-\omega_{2}}\left(\frac{e^{i \omega_{1} t}}{\omega_{1}}-\frac{e^{i \omega_{2} t}}{\omega_{2}}\right)\right] .
\end{aligned}
$$

The product of traces can be expressed in terms of the objects $T_{1}$ and $T_{4}$ defined earlier, using the fact that between $\not h \ldots \frac{1}{2}(1+\psi)$ any Dirac matrix can be decomposed into the basis $\mathbf{1}, \gamma_{5}$, $\gamma_{\perp}^{\mu}$. A straightforward calculation shows that

$$
T_{1}+T_{4}=\frac{1}{4} \operatorname{tr}\left[\gamma_{5} \frac{1+\psi}{2} \Gamma_{i} \not h \gamma_{\rho}^{\perp}\right] \cdot \operatorname{tr}\left[\gamma_{\perp}^{\rho} \not h \Gamma_{j} \frac{1+\psi}{2} \gamma_{5}\right]
$$


Taking the Fourier transform of (1), we then obtain

$$
f_{u}(\omega)=f_{v}(\omega)=-\varepsilon \frac{f_{B}^{2} m_{B}}{2} g(\omega)
$$

where

$$
g(\omega)=\delta(\bar{\Lambda}-\omega)\left[\int_{0}^{\infty} d \omega^{\prime} \frac{\phi_{+}\left(\omega^{\prime}\right)}{\omega^{\prime}}\right]^{2}+\frac{2 \phi_{+}(\bar{\Lambda}-\omega)}{\bar{\Lambda}-\omega} \mathrm{P} \int_{0}^{\infty} d \omega^{\prime} \frac{\phi_{+}\left(\omega^{\prime}\right)}{\bar{\Lambda}-\omega-\omega^{\prime}} .
$$

"P" denotes the principal-value prescription. The function $g$ has support on the half-interval $-\infty<\omega \leq \bar{\Lambda}$, as is indeed required for all shape functions [1, 2]. Its first few moments are

$$
\int d \omega g(\omega)=0, \quad \int d \omega \omega g(\omega)=0, \quad \int d \omega \omega^{2} g(\omega)=1 .
$$

The first two conditions ensure that the subleading shape functions $f_{u}$ and $f_{v}$ have vanishing norm and first moment, as required on general grounds 9 .

In order to have some explicit models at hand, we adopt two forms of the $B$-meson lightcone distribution amplitude motivated by QCD sum rules [19, 20], namely

$$
\begin{aligned}
\phi_{+}^{\mathrm{GN}}(\omega) & =\frac{\omega}{\omega_{0}^{2}} e^{-\omega / \omega_{0}} ; & \omega_{0} & =\frac{2}{3} \bar{\Lambda}, \\
\phi_{+}^{\mathrm{BIK}}(\omega) & =\frac{2 \omega_{0}^{2} \omega}{\left(\omega^{2}+\omega_{0}^{2}\right)^{2}} ; & \omega_{0} & =\frac{8}{3 \pi} \bar{\Lambda},
\end{aligned}
$$

where the relation between $\omega_{0}$ and $\bar{\Lambda}$ is implied by the equations of motion. Introducing the dimensionless variable $z=(\bar{\Lambda}-\omega) / \omega_{0}=\hat{\omega} / \omega_{0} \geq 0$, we obtain

$$
\begin{aligned}
g^{\mathrm{GN}}(\omega) & =\frac{1}{\omega_{0}^{3}}\left[\delta(z)-2 e^{-z}+2 z e^{-2 z} \operatorname{Ei}(z)\right] \\
g^{\mathrm{BIK}}(\omega) & =\frac{1}{\omega_{0}^{3}}\left[\frac{\pi^{2}}{4} \delta(z)+\frac{8}{\left(1+z^{2}\right)^{4}}\left(z \ln z+\frac{z}{2}\left(1+z^{2}\right)-\frac{\pi}{4}\left(1-z^{2}\right)\right)\right],
\end{aligned}
$$

where $\operatorname{Ei}(z)=-\mathrm{P} \int_{-z}^{\infty} d t e^{-t} / t$ is the exponential-integral function. The variable $\hat{\omega}=\bar{\Lambda}-\omega$ is the most convenient one when calculating decay spectra, because it is independent of the definition used for the $b$-quark mass [5]. A graphical representation of these two functions is shown in Figure 1. Notice the rapid fall-off of the model functions for values $\omega$ away from the endpoint. For the first model, this reflects the assumed exponential fall-off of the distribution amplitude $\phi_{+}$. But even in the second model, for which $\phi_{+}$has only a power-like fall-off, the function $g$ decreases quickly in magnitude.

So far, we have assumed that the light quark $q$ is contracted with the spectator quark in the $B$ meson. But this is not always possible (e.g., if they have different flavor), and one may entertain the possibility that the light-quark pair in the operator $O_{4 q}$ is produced from the vacuum. In this case, a possible factorization model is to write

$$
\begin{aligned}
& \left\langle\bar{B}(v)\left|(\bar{h} S)_{0} \Gamma_{1} t_{A}\left(S^{\dagger} q\right)_{t_{1} n}(\bar{q} S)_{t_{2} n} \Gamma_{2} t_{A}\left(S^{\dagger} h\right)_{t n}\right| \bar{B}(v)\right\rangle \\
& \approx \mathcal{B}\left\langle\bar{B}(v)\left|\left[(\bar{h} S)_{0} \Gamma_{1} t_{A}\right]_{\alpha}^{i}\left[\Gamma_{2} t_{A}\left(S^{\dagger} h\right)_{t n}\right]_{\beta}^{j}\right| \bar{B}(v)\right\rangle\left\langle 0\left|\left[\left(S^{\dagger} q\right)_{t_{1} n}\right]_{\alpha}^{i}\left[(\bar{q} S)_{t_{2} n}\right]_{\beta}^{j}\right| 0\right\rangle
\end{aligned}
$$



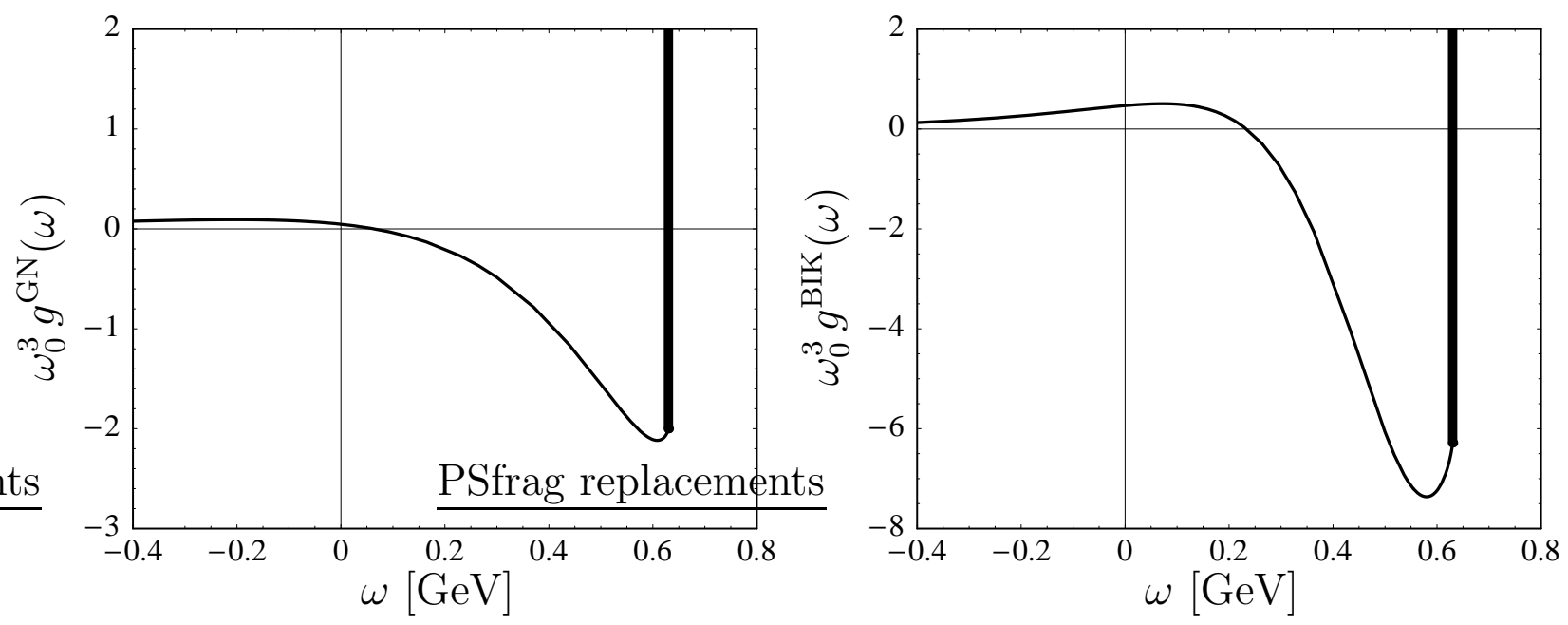

Figure 1: Model predictions for the function $g(\omega)$ obtained using $\bar{\Lambda}=0.63 \mathrm{GeV}$, corresponding to $m_{b}=4.65 \mathrm{GeV}$. The thick lines represent the $\delta$-function localized at $\omega=\bar{\Lambda}$.

where $\mathcal{B}$ is a bag parameter, and we have left the Dirac structures arbitrary for the moment. Lorentz and gauge invariance dictate that

$$
\left\langle 0\left|\left[\left(S^{\dagger} q\right)_{t_{1} n}\right]_{\alpha}^{i}\left[(\bar{q} S)_{t_{2} n}\right]_{\beta}^{j}\right| 0\right\rangle=-\frac{\delta^{i j}}{4 N_{c}}\langle\bar{q} q\rangle\left[\mathbf{1}+i \Lambda_{h}\left(t_{2}-t_{1}\right) \not h\right]_{\alpha \beta},
$$

where $\langle\bar{q} q\rangle$ is the local quark condensate, and $\Lambda_{h}$ is a hadronic parameter. From above, we now obtain in our model

$$
\begin{aligned}
\left\langle\bar{B}(v)\left|(\bar{h} S)_{0} \Gamma_{1} t_{A}\left(S^{\dagger} q\right)_{t_{1} n}(\bar{q} S)_{t_{2} n} \Gamma_{2} t_{A}\left(S^{\dagger} h\right)_{t n}\right| \bar{B}(v)\right\rangle \\
=-\frac{\mathcal{B} C_{F}}{4 N_{c}}\langle\bar{q} q\rangle\left[\left\langle\bar{B}(v)\left|\bar{h}(0)[0, t] \Gamma_{1} \Gamma_{2} h(t n)\right| \bar{B}(v)\right\rangle\right. \\
\left.\quad+i \Lambda_{h}\left(t_{2}-t_{1}\right)\left\langle\bar{B}(v)\left|\bar{h}(0)[0, t] \Gamma_{1} \not h \Gamma_{2} h(t n)\right| \bar{B}(v)\right\rangle\right] .
\end{aligned}
$$

Applying this formula to the specific case of the operator in (11), for which $\Gamma_{1}=\Gamma_{i} \not h \gamma_{\rho}^{\perp}$ and $\Gamma_{2}=\gamma_{\perp}^{\rho} \not h \Gamma_{j}$, we see that the Dirac structures $\Gamma_{1} \Gamma_{2}$ and $\Gamma_{1} \not h \Gamma_{2}$ both vanish due to $\not k^{2}=0$. Therefore, the non-valence contributions vanish in the vacuum-insertion approximation.

Let us briefly discuss the phenomenological implications of our results by considering three decay spectra in semileptonic $\bar{B} \rightarrow X_{u} l^{-} \bar{\nu}$ decay, referring to [9] for details and derivations. In all cases we show the contributions of the leading-order shape function $\hat{S}(\hat{\omega})$ and of the fourquark shape functions as calculated in our model, ignoring other subleading shape-function contributions, which have already been discussed in [9]. In our model, only decay distributions of charged $B$ mesons are effected by the four-quark contributions. Of particular interest for a measurement of $\left|V_{u b}\right|$ are the spectra in the variables $E_{l}$ (charged-lepton energy), $P_{+}$(hadronic energy minus momentum), and $s_{H}$ (hadronic invariant mass squared). The corresponding 
normalized distributions are given by

$$
\begin{aligned}
& \frac{1}{\Gamma} \frac{d \Gamma}{d E_{l}}=4 \int_{0}^{m_{B}-2 E_{l}} \frac{d \hat{\omega}}{m_{B}-\hat{\omega}}\left[\hat{S}(\hat{\omega})+3 \varepsilon \pi \alpha_{s} f_{B}^{2} \hat{g}(\hat{\omega})\right], \\
& \frac{1}{\Gamma} \frac{d \Gamma}{d P_{+}}=\hat{S}\left(P_{+}\right)+\frac{2}{3} \varepsilon \pi \alpha_{s} f_{B}^{2} \hat{g}\left(P_{+}\right), \\
& \frac{1}{\Gamma} \frac{d \Gamma}{d s_{H}}=\frac{1}{m_{B}} \int_{s_{H} / m_{B}}^{\infty} \frac{d \hat{\omega}}{\hat{\omega}} F\left(\hat{\omega}, \frac{s_{H}}{m_{B} \hat{\omega}}\right),
\end{aligned}
$$

where $\hat{g}(\hat{\omega})=g(\bar{\Lambda}-\hat{\omega})$, and in the latter case

$$
F(\hat{\omega}, r)=2 r^{2}(3-2 r) \hat{S}(\hat{\omega})+4 r(1-r) \varepsilon \pi \alpha_{s} f_{B}^{2} \hat{g}(\hat{\omega}) .
$$

Note that the contribution to the charged-lepton spectrum is much larger than that in the other two cases. We have studied the numerical impact of the four-quark contributions to these spectra using the model functions in (14) with $\bar{\Lambda}=0.63 \mathrm{GeV}$ and the default choice for the leading-order shape function from [9]. We take $f_{B}=200 \mathrm{MeV}$ for the $B$-meson decay constant and $\alpha_{s}=0.3$ for the strong coupling at the intermediate scale. Even without assuming a significant color suppression the effects are very small. For $\varepsilon=1$, sizable distortions of the $P_{+}$and $s_{H}$ spectra occur below $P_{+} \approx 0.5 \mathrm{GeV}$ and $s_{H} \approx 1.5 \mathrm{GeV}^{2}$, while those of the chargedlepton energy spectrum are located above $E_{l} \approx 2.2 \mathrm{GeV}$. For more realistic values $|\varepsilon| \ll 1$, the effects are even smaller. Varying the input parameters within reasonable limits does not change this conclusion. The four-quark contributions are proportional to the combination $\varepsilon \pi \alpha_{s} f_{B}^{2} / \bar{\Lambda}^{3}$ times a dimensionless function of the ratio $\hat{\omega} / \bar{\Lambda}$. Assuming $f_{B}=(200 \pm 30) \mathrm{MeV}$ and $\bar{\Lambda}=(0.63 \pm 0.07) \mathrm{GeV}$, we have $\varepsilon \pi \alpha_{s} f_{B}^{2} / \bar{\Lambda}^{3}=(0.15 \pm 0.07) \varepsilon \mathrm{GeV}^{-1}$, where the parametric uncertainty can be absorbed into our ignorance about the color-suppression factor $\varepsilon$.

Figure 2 shows results for the partial rate fractions obtained by integrating the spectra in (18) over $E_{l} \geq E_{0}=\left(m_{B}-\Delta_{E}\right) / 2, P_{+} \leq \Delta_{P}$, and $s_{H} \leq s_{0}=m_{B} \Delta_{M}$, where the quantities $\Delta_{i}$ are chosen such that in all three cases the charm background starts at $\Delta_{i}=$ $m_{D}^{2} / m_{B} \approx 0.66 \mathrm{GeV}$. The effects of the four-quark shape functions are included under the extreme assumption of no color suppression $(\varepsilon=1)$. In reality, we expect their effects to be significantly smaller. If we consider the partial rate fractions obtained by integrating the spectra over the phase space not contaminated by charm background, we find that the largest effects in the two models lead to

$$
F_{E_{l}}=(6.5+1.4 \varepsilon) \%, \quad F_{P_{+}}=(60.9-2.3 \varepsilon) \%, \quad F_{s_{H}}=(80.6-0.7 \varepsilon) \% .
$$

In the latter two cases these corrections are negligible even if the color-suppression factor $\varepsilon$ is not particularly small.

For comparison, we note that the authors of [8] estimate (using naive dimensional analysis, and ignoring color suppression) that the impact of four-quark shape functions on the chargedlepton energy fraction could be as large as $180 \%$ of the leading term. Even for $\varepsilon=1$ this 

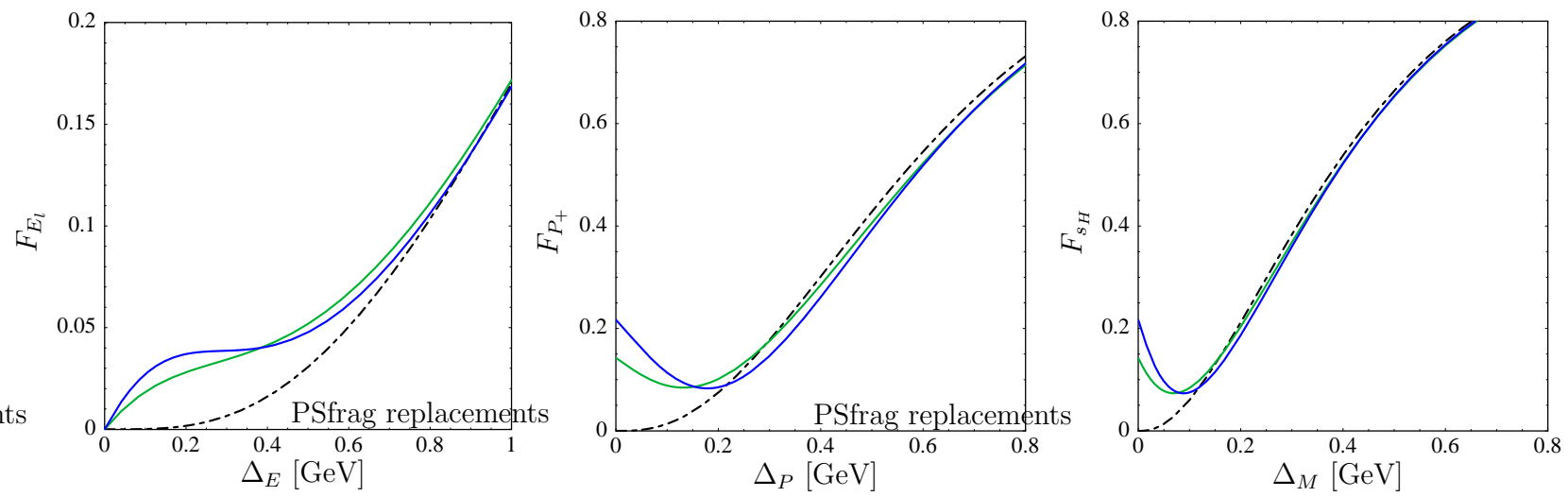

Figure 2: Model predictions for the partial $\bar{B} \rightarrow X_{u} l^{-} \bar{\nu}$ rate fractions with cuts on $E_{l}, P_{+}$, and $s_{H}$, respectively. The dashed line shows the leading-order result, while the two solid lines include the contributions from four-quark shape functions, evaluated setting $\varepsilon=1$.

would be an order of magnitude bigger than our estimate. The main reason is that these authors include an "enhancement factor" $g_{s}^{2}=4 \pi \alpha_{s} \approx 4$ in their estimate of the powersuppressed effects, as opposed to, say, $\pi \alpha_{s} \approx 1$. Clearly, dimensional analysis cannot tell the difference between these two factors. The precise numerical coefficients multiplying the fourquark shape-function contributions to a given observable can only be determined by explicit calculation, as done in (18). Note that following the reasoning of [8] one would associate an enhancement factor $g_{s}^{2}=4 \pi \alpha_{s} \sim 12$ with soft-gluon exchange (for which $\alpha_{s} \sim 1$ ), which would be wrong, since the corresponding powers of $g_{s}$ are already included in what is conventionally called $\Lambda_{\mathrm{QCD}}$.

In summary, we have presented a simple, but well-motivated model for the four-quark subleading shape functions $f_{u}(\omega)$ and $f_{v}(\omega)$, which contribute at order $\Lambda_{\mathrm{QCD}} / m_{b}$ to inclusive $B$-decay spectra in the endpoint region. We have shown that in the vacuum-insertion approximation non-zero contributions only arise from four-quark operators for which the light quark flavor matches that of the $B$-meson spectator quark. Vacuum pair production of the light quark pair, though not suppressed on general grounds, does not contribute due to the Dirac structure of the relevant operators. The result for the subleading shape functions $f_{u}$ and $f_{v}$ is given by a color-suppression factor times a double convolution integral over a product of two $B$-meson light-cone distribution amplitudes. Using two simple models for this function, we have obtained explicit forms for the subleading shape functions, which are compatible with all known constraints from analyticity and moment relations. The corresponding impact on the decay distributions in semileptonic $\bar{B} \rightarrow X_{u} l^{-} \bar{\nu}$ decays is found to be negligible. While the results reported here are admittedly model dependent, we believe that they support our earlier claim [9] that these four-quark contributions are likely to be smaller than other subleading shape-function effects.

Acknowledgments: I am grateful to Björn Lange for useful discussions. This research was supported by the National Science Foundation under Grant PHY-0355005, and by the Department of Energy under Grant DE-FG02-90ER40542. 


\section{References}

[1] M. Neubert, Phys. Rev. D 49, 3392 (1994) hep-ph/9311325; Phys. Rev. D 49, 4623 (1994) hep-ph/9312311.

[2] I. I. Y. Bigi, M. A. Shifman, N. G. Uraltsev and A. I. Vainshtein, Int. J. Mod. Phys. A 9, 2467 (1994) hep-ph/9312359.

[3] G. P. Korchemsky and G. Sterman, Phys. Lett. B 340, 96 (1994) hep-ph/9407344.

[4] C. W. Bauer and A. V. Manohar, Phys. Rev. D 70, 034024 (2004) hep-ph/0312109.

[5] S. W. Bosch, B. O. Lange, M. Neubert and G. Paz, Nucl. Phys. B 699, 335 (2004) hep-ph/0402094; M. Neubert, Eur. Phys. J. C 40, 165 (2005) hep-ph/0408179.

[6] C. W. Bauer, S. Fleming, D. Pirjol and I. W. Stewart, Phys. Rev. D 63, 114020 (2001) hep-ph/0011336; C. W. Bauer, D. Pirjol and I. W. Stewart, Phys. Rev. D 65, 054022 (2002) hep-ph/0109045.

[7] M. Beneke, A. P. Chapovsky, M. Diehl and T. Feldmann, Nucl. Phys. B 643, 431 (2002) hep-ph/0206152; M. Beneke and T. Feldmann, Phys. Lett. B 553, 267 (2003) hep-ph/0211358.

[8] K. S. M. Lee and I. W. Stewart, Nucl. Phys. B 721, 325 (2005) hep-ph/0409045.

[9] S. W. Bosch, M. Neubert and G. Paz, JHEP 0411, 073 (2004) hep-ph/0409115.

[10] M. Neubert, Phys. Rept. 245, 259 (1994) hep-ph/9306320.

[11] C. W. Bauer, M. E. Luke and T. Mannel, Phys. Rev. D 68, 094001 (2003) hep-ph/0102089; Phys. Lett. B 543, 261 (2002) hep-ph/0205150.

[12] A. K. Leibovich, Z. Ligeti and M. B. Wise, Phys. Lett. B 539, 242 (2002) hep-ph/0205148.

[13] C. N. Burrell, M. E. Luke and A. R. Williamson, Phys. Rev. D 69, 074015 (2004) hep-ph/0312366.

[14] I. I. Y. Bigi, B. Blok, M. A. Shifman, N. Uraltsev and A. I. Vainshtein, hep-ph/9401298.

[15] M. Neubert and C. T. Sachrajda, Nucl. Phys. B 483, 339 (1997) hep-ph/9603202.

[16] M. Di Pierro and C. T. Sachrajda, Nucl. Phys. B 534, 373 (1998) hep-lat/9805028.

[17] D. Becirevic, hep-ph/0110124.

[18] M. S. Baek, J. Lee, C. Liu and H. S. Song, Phys. Rev. D 57, 4091 (1998) hep-ph/9709386.

[19] A. G. Grozin and M. Neubert, Phys. Rev. D 55, 272 (1997) hep-ph/9607366.

[20] V. M. Braun, D. Y. Ivanov and G. P. Korchemsky, Phys. Rev. D 69, 034014 (2004) hep-ph/0309330. 